\title{
Vector capacity of Anopheles sinensis in malaria outbreak areas of central China
}

\author{
Jia-Yun Pan ${ }^{1,2}$, Shui-Sen Zhou ${ }^{1,2^{*}}$, Xiang Zheng ${ }^{1,2}$, Fang Huang ${ }^{1,2}$, Duo-Quan Wang ${ }^{1,2}$, Yu-Zu Shen ${ }^{3}$, Yun-Pu Su ${ }^{4}$, \\ Guang-Chao Zhou ${ }^{5}$, Feng Liu ${ }^{6}$ and Jing-Jing Jiang ${ }^{3}$
}

\begin{abstract}
Background: Both falciparum and vivax malaria were historically prevalent in China with high incidence. With the control efforts, the annual incidence in the whole country has reduced to $0.0001 \%$ except in some areas in the southern borders after 2000. Despite this, the re-emergence or outbreak of malaria was unavoidable in central China during 2005-2007. In order to understand the role of the vector in the transmission of malaria during the outbreak period, the vector capacity of An. sinensis in Huanghuai valley of central China was investigated.
\end{abstract}

Findings: The study was undertaken in two sites, namely Huaiyuan county of Anhui province and Yongcheng county of Henan province. In each county, malaria cases were recorded for recent years, and transmission risk factors for each study village including anti-mosquito facilities and total number of livestock were recorded by visiting each household in the study sites. The specimens of mosquitoes were collected in two villages, and population density and species in each study site were recorded after the identification of different species, and the blood-fed mosquitoes were tested by ring precipitation test. Finally, various indicators were calculated to estimate vector capacity or dynamics, including mosquito biting rate (MBR), human blood index (HBI), and the parous rates (M). Finally, the vector capacity, as an important indicator of malaria transmission to predict the potential recurrence of malaria, was estimated and compared in each study site.

About $93.0 \%$ of 80 households in Huaiyuan and $89.3 \%$ of 192 households in Yongcheng had anti-mosquito facilities. No cattle or pigs were found, only less than 10 sheep were found in each study village. A total of 94 and 107 Anopheles spp. mosquitos were captured in two study sites, respectively, and all of An. sinensis were morphologically identified. It was found that mosquito blood-feeding peak was between 9:00 pm and 12:00 pm. Man biting rate of An. sinensis was 6.0957 and 5.8621 (mosquitoes/people/night) estimated by using half-night human bait trap method and full-capture method, respectively. Human blood indexes (HBI) were 0.6667 (6/9) and 0.6429 (18/28), and man-biting habits were 0.2667 and 0.2572 in two sites, respectively. Therefore, the expectation of infective life and vector capacity of An. sinensis was 0.3649-0.4761 and 0.5502-0.7740, respectively, in Huanhuai valley of central China where the outbreak occurred, which is much higher than that in the previous years without malaria outbreak.

Conclusions: This study suggests that vivax malaria outbreak in Huanhuai valley is highly related to the enhancement in vector capacity of An. sinensis for P. vivax, which is attributed to the local residents' habits and the remarkable drop in the number of large livestock leading to disappearance of traditional biological barriers.

Keywords: Vector capacity, Anopheles sinensis, Outbreak, Malaria, Plasmodium vivax, China

\footnotetext{
* Correspondence: ccdczss@sh163.net

${ }^{1}$ National Institute of Parasitic Diseases, Chinese Center for Disease Control and Prevention, Shanghai 200025 People's Republic of China

${ }^{2}$ WHO Collaborating Centre for Malaria, Schistosomiasis and Filariasis, Key Laboratory of Parasite \& Vector Biology, Ministry of Health, Shanghai 200025

People's Republic of China

Full list of author information is available at the end of the article
}

\section{Biomed Central}

(C) 2012 Pan et al.; licensee BioMed Central Ltd. This is an Open Access article distributed under the terms of the Creative Commons Attribution License (http://creativecommons.org/licenses/by/2.0), which permits unrestricted use, distribution, and reproduction in any medium, provided the original work is properly cited. 


\section{Background}

Malaria is one of the most important parasitic diseases in People's Republic of China [1-3]. Historically, a higher incidence of malaria, caused by infection of both Plasmodium vivax and P. falciparum, was observed among human populations in central China, such as Anhui, Henan, Hubei, Shangdong and Jiangsu provinces, located in Huanghuai valley $[2,4]$. Two big epidemics of malaria occurred at the beginning of the 1960s and the 1970s, of which the incidence rate was $1.55 \%$ and $2.96 \%$, respectively [5]. Following the great control efforts, the incidences of both species of malaria had reduced remarkably. For instance, only $0.1 \%$ to $0.0017 \%$ of annual malaria incidence was reported during the 1980s and the 1990s in the country, respectively [6]. While, the incidence of vivax malaria transmitted by Anopheles sinensis was increased after 2000, due to the social and environment changes in macro scenario, such as population mobility increase due to the development of the economy, global warming, leading to the longer transmission season for $P$. vivax, and so on [4,7-10]. About $86 \%$ of malaria cases in the country were located in the Huanghuai valley of central China where the predominant vector mosquito was $A n$. sinensis [2,11,12]. In this region, most of the outbreaks that occurred were localized at village level or township level of 4 provinces in central China, including Anhui, Henan, Hubei and Jiangsu provinces [13,14]. Previous studies in central China have shown that vector capacity is one of most important factors contributing to the transmission of vivax malaria, which could accelerate re-emergence of malaria outbreaks [2,15-18], but only a few relevant field-epidemiological investigations on the re-emergence of vivax malaria have been reported in China as well as in the Greater Mekong Subregion $[19,20]$.

China launched the National Malaria Elimination Program (NMEP) in 2010, with its goal to eliminate malaria in China by 2020 [21,22]. In the NMEP, vector control is one of the important components in rapid response to the malaria transmission in outbreak foci as well as to improve the efficiency of case management, which has been promoted by both World Health Organization (WHO) and Roll Back Malaria Partnership (RBM) for reduction of malaria transmission [23,24]. One of the most difficult issues in the elimination process is to have real-time surveillance and response systems to monitor the changes of transmission patterns in order to guide the elimination efforts in the high risk areas [8]. It is believed that transmission intensity of human malaria is highly dependent on the vector capacity and competence of local mosquitoes. Recent research aimed at understanding the relationship between vector capacity and the transmission patterns of malaria is of significant concern $[25,26]$. Therefore, it is important to understand the changes of

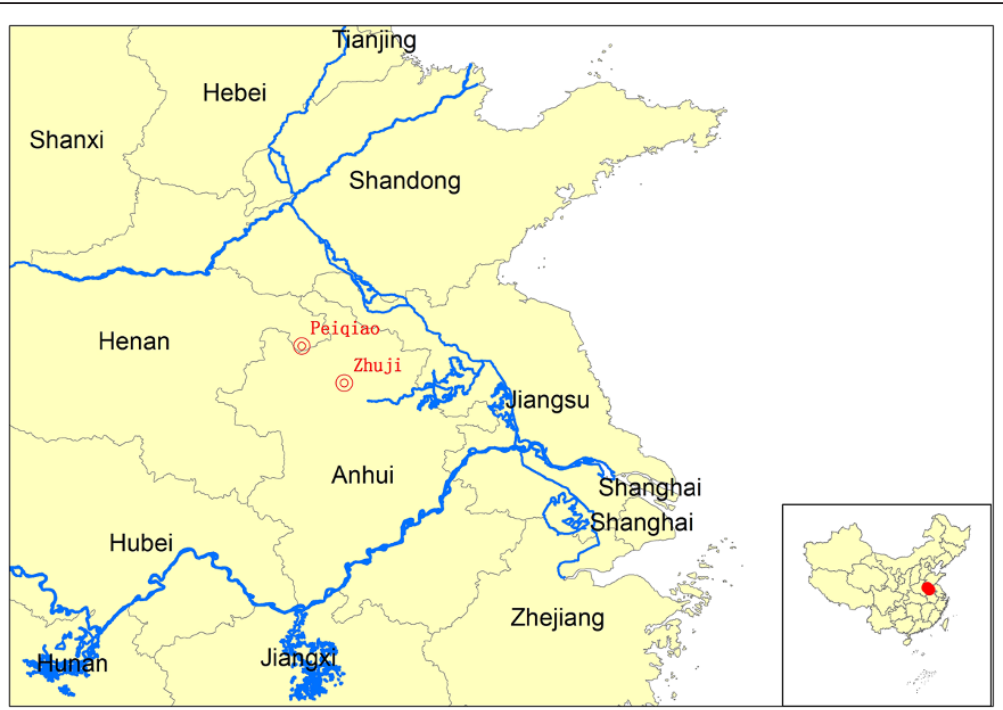

Figure 1 Location of the two study sites. One study site was located in the Zhao village, Zhuji township, Huaiyuan county ( $33^{\circ} 14^{\prime} \mathrm{N}$ and $116^{\circ}$ $\left.51^{\prime} \mathrm{E}\right)$, Anhui province (site A), the other one was located in Pengguanyao village, Peiqiao township, Yongcheng county $\left(33^{\circ} 48^{\prime} \mathrm{N}\right.$ and $\left.116^{\circ} 12^{\prime} \mathrm{E}\right)$, Henan province. 
An. sinensis vector capacity in the high risk areas or in outbreak foci. But few investigations have explored the changes in vector capacity of $A n$. sinensis at the micro settings, although it is believed that the vector capacity of mosquitoes is a good indicator to assess the transmission level of vivax malaria [26-28]. It is worthwhile understanding the role of the anopheles mosquitoes in the transmission of vivax malaria in Huanghuai valley during outbreaks, which will guide the formulation of elimination strategy and establishment of surveillance and response systems in the NMEP in China.

The investigation on vector capacity of An. sinensis in two outbreak areas of Anhui and Henan provinces was carried out during summer of 2007 when vivax malaria sporadic outbreaks were still taking place, in order to clearly understand the change patterns for vector capacity of $A n$. sinensis which will promote the surveillance-response strategy [26].

\section{Methods}

\section{Area and time of investigation}

The study was undertaken in two sites, including Zhao village, Zhuji township, Huaiyuan county $\left(33^{\circ} 14^{\prime} \mathrm{N}\right.$ and $\left.116^{\circ} 51^{\prime} \mathrm{E}\right)$, Anhui province, and Pengguanyao village, Peiqiao township, Yongcheng county $\left(33^{\circ} 48^{\prime} \mathrm{N}\right.$ and $116^{\circ}$ $\left.12^{\prime} \mathrm{E}\right)$, Henan province (Figure 1). Both counties are located in the plain region, surrounded with rice pads, and planted with crops, such as beans, rice etc., and chemical compounds are rarely used as insecticides. The drainage system is not adequate, facilitated with more open water around villages in Summer time, thus providing more suitable habitats for the development of An. sinensis larvae.

The investigation was carried out at two time points, e.g. early August and late August, 2007, respectively, with an interval of 10 days, during the peak transmission period of vivax malaria and its sporadic outbreaks which still occurred in the study region. Half-night mosquito biting experiments were undertaken twice in Huaiyuan county and Yongcheng county, respectively. Full-capture method was used to collect Anopheles spp. mosquito specimens in the early morning inside and outside of mosquito-nets in human living rooms, as well as in livestock rooms in the study sites.

\section{Malaria incidence and risk factors}

All reported data on malaria cases recorded by the Center for Disease Control and Prevention in each county was collected, and the malaria incidence in each study county was estimated in 2007 and compared with reported data from the region in previous years. Malaria transmission-related risk factors for each study site, including usage of antimosquitofacilities and total number of livestock, were recorded by visiting each household of the study villages.

\section{Vector capacity}

The specimens of mosquito were collected in two villages, and population density and species in each study site were recorded after the identification of different species, based on morphology of mosquitoes, and the saturated mosquitoes were tested by ring precipitation test [29]. Finally, various indicators were calculated to estimate vector capacity, including mosquito biting rate (MBR), human blood index (HBI), and the parous rates $(\mathrm{M})[30,31]$ using the following details of methodology.

The vector capacity was estimated from the calculation of three indices [32]. Firstly, the investigation on mosquito biting rate was conducted by two approaches. One approach is the half-night bite rate, which was carried out during 19:30 pm to 24:00 pm by the human attraction method, this was carried out 4 times to capture as many anopheles mosquitoes as possible [29]. Briefly, a person was inside the half-opened mosquito-net, and Anopheles spp. mosquitoes were continuously collected over the time period [12]. The number of An. sinensis collected was recorded. The half-night man biting rate was calculated by following formula:

$$
\begin{aligned}
& \text { Half }- \text { night man biting rate (No. mosquitoes/people) } \\
& =\frac{\text { number of mosquitoes captured inside and outside the net }}{\text { number of people for bait trap inside net }}
\end{aligned}
$$

The other approach used to investigate mosquito biting rate was performed in the same villages using a full-capture method [33]. Briefly, Anopheles spp. mosquitoes were searched for and captured in the early morning during 6:00 am to 7:00 am. All Anopheles spp. mosquitoes were captured inside each mosquito-net in houses, and barnyards in the investigation village, covering a population of 300-450 people for each village. The number of $A n$. sinensis captured was recorded, and the mosquito man-biting rate per night was calculated by the following formula:

\section{Man biting density inside net whole night (No. mosquitoes/people) $=\frac{\text { the number of mosquitoes captured from nets }}{\text { the number of people in the nets surveyed }}$}

And then,

Man-biting rate (ma) (No. mosquitoes/ people/ night $)=$ half-night man biting rate + Man biting density inside net whole night.

Secondly, the human blood index (HBI) was estimated using the method of ring precipitation test [29]. All stomach blood specimens were examined from the 
captured Anopheles spp. mosquitoes engorged with blood after these mosquitoes had been morphologically identified. A serologic ring precipitation test was conducted to estimate the HBI, both HBI and manbiting habit (a) were calculated by using the following formula [33]:

$$
\begin{aligned}
& \text { HBI }=\frac{\text { No. mosquitoes of the human blood }}{\text { No. mosquitoes saturated }} \\
& \text { Man }- \text { biting habit }(\mathrm{a}) \\
& \quad=\frac{\mathrm{HBI}}{\text { Gonotrophic cycle of An. sinensis }(\mathrm{x}=2.5 \text { days })}
\end{aligned}
$$

Thirdly, the parous rate (M) was estimated. Briefly, egg-laying mosquitoes were distinguished by dissecting ovaries to see the morpho-rami of ovarial trachea, and $x$ stands for the period in days from human biting to laying eggs, which will be easily considered as the gonotrophic cycle period, e.g. An. sinensis will take about 2.5 days to finish the gonotrophic cycle. Then, the parous rate (M) and daily survival rate $\left(p=M^{1 / x}\right)$ were estimated. Given the local average temperatures and the sporgenous cycle (n) of 10 days [31,34], we estimated the expectation of infective life of An. sinensis $\left(\mathrm{p}^{\mathrm{n}} /-\ln \mathrm{P}\right)$.

Finally, the vector capacity (C) [35], an important indicator and measure of malaria transmission predicting recurrence of transmission [29,35-37], was estimated by using the following formula [29]:

$$
\text { Vector capacity }(C)=\frac{\mathrm{ma}^{2} \mathrm{P}^{\mathrm{n}}}{-\ln \mathrm{P}}
$$

Where $\mathrm{P}$ is survival rate of mosquitoes, $\mathrm{n}$ is the mean days for reproduction of Plasmodium sporozoites in mosquitoes under the local temperature.

\section{Ethical considerations}

The study protocol was approved by the institutional review board of the National Institute of Parasitic Diseases, Chinese Center for Disease Control and Prevention in Shanghai. The objectives, procedures, and potential risks of the mosquito biting experiment using the human attraction method were carefully explained to each participant. Interested individuals provided written informed consent in person before participation in the study.

\section{Results}

The malaria incidence and risk factors

The incidence of malaria in 2007 were $9.1 / 10^{4}$ (1167/ $1287000)$ and $22.2 / 10^{4}(2883 / 1297000)$ in Huaiyuan county, Anhui province and Yongcheng county, Henan province, respectively.

A total of 343 residents from 86 households in Huaiyuan, and 860 residents from 215 households in Yongcheng were visited. About $93.0 \%$ of the households $(80 / 86)$ in Huaiyuan and $89.3 \%$ of the households (192/ 215) in Yongcheng had anti-mosquito facilities, such as mosquito nets, screened windows, screened doors and mosquito repellent. On average, about 1.3 (51/39) and $1.7(219 / 129)$ people were used for one mosquito-net, and their mosquito-net coverage rates per household were $45.34 \%$ and $60.0 \%$ in Huaiyuan and Yongcheng, respectively. Neither cattle nor pigs were found in the study villages of two counties, only 9 and 7 sheep free-roaming were found in Huaiyuan and Yongcheng, respectively.

\section{Vector capacity}

\section{Anopheles populations and its blood-sucking behavior}

The half-night human bait trap was performed twice in Huaiyuan county and Yongcheng county, respectively. A total of 201 Anopheles spp. mosquitoes were captured in

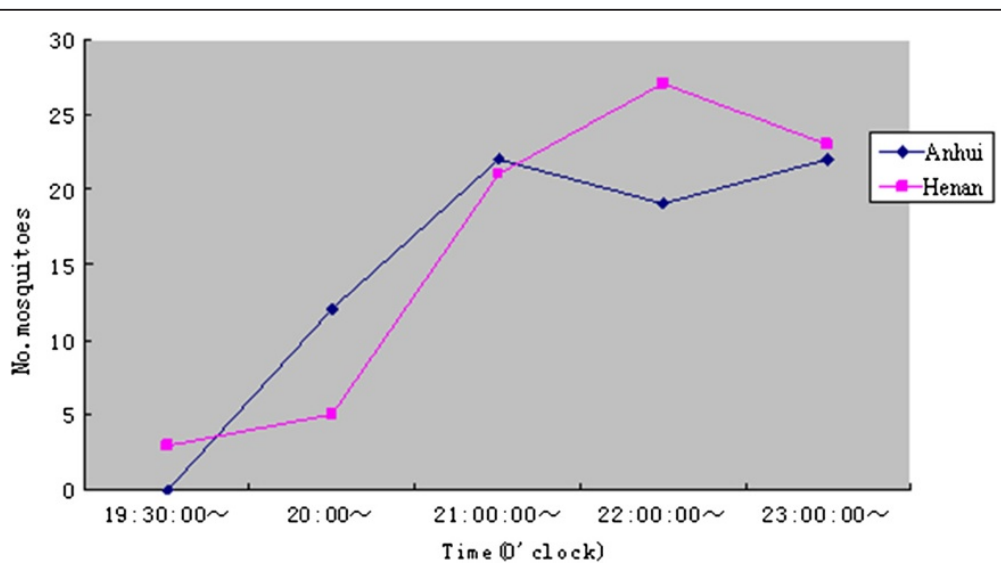

Figure 2 Patterns of blood feeding activities of Anopheles sinesis at night. Both curves indicating patterns of mosquito blood feeding activities from Anhui (blue line) and Henan (pink line) provinces. 
study sites, among them 94 were found in Huaiyuan and 107 were collected in Yongcheng. Morphological analysis showed that they all belonged to species of Ansinensis. Results from the half-night human bait trap showed that An. sinensis activity for taking blood meals occurred from 7:30 pm to $12: 00 \mathrm{pm}$, and the blood-feeding peak was between 9:00 pm and 12:00 pm (Figure 2).

The mosquito specimens were collected by the fullcapture method 3 times in total in the study sites. A total of 16 and 3 An. sinensis mosquitoes were obtained from 39 mosquito-nets and 3 livestock rooms in Huaiyuan county, respectively. 16 and 12 An. sinensis mosquitoes were collected from 42 mosquito-nets and 3 livestock rooms in Yongcheng, respectively. It was estimated that the man biting rate from the study sites was 6.0957 (mosquitoes/people/night) and 5.8621 (mosquitoes/people/night) by using the half-night human bait trap method and full-capture method, respectively (Table 1, Figure 3).

\section{Vector capacity}

A total of 47 An. sinensis mosquitoes were captured inside the houses, mosquito-nets and sheep yards in the study sites. Among them, 9 out of 19 An. sinensis mosquitoes engorged with blood were captured in Huaiyuan, and 28 out of 28 An. sinensis mosquitoes engorged with blood were captured in Yongcheng. Ring precipitation tests showed that HBI of these two study sites were $0.6667(6 / 9)$ and $0.6429(18 / 28)$, thus the man-biting habits (a) were 0.2667 and 0.2572 in Huaiyuan and Yongcheng, respectively.

A total of 93 An.sinensis were captured and dissected for examination of ovaries. The parous rates $(\mathrm{M})$ were $57.14 \%(28 / 49)$ and $54.55 \%(24 / 44)$ from Huaiyuan and Yongcheng, respectively. Given the local average temperatures, the sporgenous cycle of An. sinensis was 10 days and the gonotrophic cycle was 2.5 days [38], we estimated that the expectations of infective life of An. sinensis were 0.4761 and 0.3649 from Huaiyuan and Yongcheng, respectively. Therefore, the vectorial capacities of An. sinensis in Huaiyuan and Yongcheng were 0.7740 and 0.5502 , respectively (Table 2). When compared with the vector capacity of An. sinensis from the reported data in the previous years (1984-1994), the three indicators including the man-biting habit, human blood index and vector capacity were changed

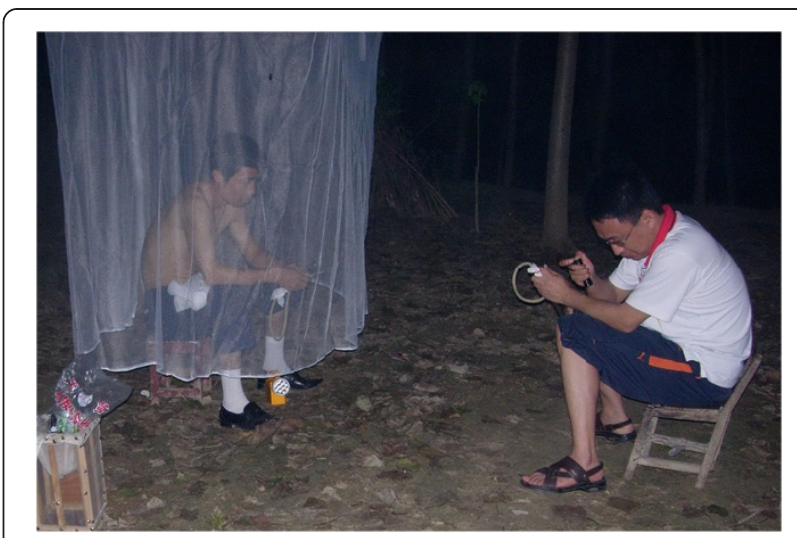

Figure 3 A photograph of Anopheles sinesis mosquito capture at night, using half-night human bait trap method inside mosquito net.

in consistency with the fluctuations of malaria incidence during the same period. The significant changes in man-biting habit reflected that the number of livestock as sources of infection changed during the study period as well, indicating that this change in pattern is closely related to the reduction of livestock numbers in the study sites.

\section{Discussion}

Vector control has been one of the important components for the malaria control program leading to elimination [39]. There are various methods of vector control that are used in blocking the transmission of P. vivax [28]. While the level of $P$. vivax transmission is significantly related to two main factors, such as vector capacity and the number of infectious sources or human cases [25]. When the environmental conditions are appropriate, malaria outbreaks could occur, involving large numbers of human cases $[4,40]$.

In terms of infectious sources, human cases infected with $P$. vivax have been reduced significantly in the Huanghuai valley of China in the new millennium, although it was more than 10 million cases per year in the 1950s. In particular, following the large-scale efforts of the NMCP in China since the 1970s, malaria incidence in the areas where $A n$. sinensis was the only vector had been reduced significantly to a low level in the 1990s. According to the national data on malaria transmission, the average annual incidences of vivax malaria in 1998 in Anhui and Henan province with An. sinensis being

Table 1 Man-biting rate estimation in study sites

\begin{tabular}{|c|c|c|c|c|c|c|}
\hline Location & Bait method & $\begin{array}{l}\text { Number of } \\
\text { bait }\end{array}$ & $\begin{array}{l}\text { Number of } \\
\text { Capture }\end{array}$ & $\begin{array}{l}\text { Half-night } \\
\text { bite rate * }\end{array}$ & $\begin{array}{l}\text { Biting rate in } \\
\text { the mosquito nets } \S\end{array}$ & $\begin{array}{l}\text { Man-biting } \\
\text { rate\# }\end{array}$ \\
\hline Huaiyuan, Anhui province & Human bait outdoor & 13 & 75 & 5.7692 & $0.3265(16 / 49)$ & 6.0957 \\
\hline Yongcheng, Henan province & Human bait indoor and outdoor & 14 & 79 & 5.6429 & $0.2192(16 / 73)$ & 5.8621 \\
\hline
\end{tabular}

Note: * Half-night bite rate was measured in mosquitoes/people; §Biting rate in the mosquito nets was measured in mosquitoes/people/night; and \#Biting rate was measured in mosquito/people/night). 
Table 2 Comparison of vector capacity of An. sinensis between outbreak time period and non outbreak time period in areas where only species of An. sinensis served as vectors of vivax malaria

\begin{tabular}{lcccccc}
\hline Time (Location) & $\begin{array}{l}\text { Incidence rate } \\
(\%)\end{array}$ & $\begin{array}{l}\text { Man-biting rate } \\
(\mathbf{m a})\end{array}$ & $\begin{array}{l}\text { Human blood } \\
\text { index (HBI) }\end{array}$ & $\begin{array}{l}\text { Man-biting } \\
\text { habit (a) }\end{array}$ & $\begin{array}{l}\text { Expectation infective life } \\
\left(\mathbf{p}^{\mathbf{n}} / \text { /-Inp) }\right.\end{array}$ & $\begin{array}{l}\text { Vector capacity } \\
(\mathbf{C}) \text { Ma } \times \mathbf{a} \times\left(\mathbf{p}^{\mathbf{n}} / \text {-Inp) }\right.\end{array}$ \\
\hline $1984 \S$ & 0.02 & 20.8 & 0.0300 & 0.0120 & 0.2424 & 0.0605 \\
$1994 \#$ & 0.01 & 4.7 & 0.128 & 0.051 & 0.7275 & 0.1744 \\
2007 (Huaiyuan) & 0.091 & 6.0957 & 0.600 & 0.2667 & 0.4761 & 0.7740 \\
2007 (Yongcheng) & 0.222 & 5.8621 & 0.666 & 0.2572 & 0.3649 & 0.5502 \\
\hline
\end{tabular}

*: Expectation of infective life was measured in days. §: Reported data in Huanghuai valley, China. \#: Reported data in Henan provinces, China.

the only vector, were below $1 / 10^{5}$ and $0.03 / 10^{5}$, respectively. However, the incidence of vivax malaria had a resurgence in Huanghuai valley after 2003 [4,41]. For example, the annual incidence of malaria from 2004 to 2006 was $101.22 / 10^{5}, 95.38 / 10^{5}$ and $90.67 / 10^{5}$ in Anhui province, and was $12.29 / 10^{5}, 54.99 / 10^{5}$ and $222.82 / 10^{5}$ in Henan province, respectively, which were significantly higher than those recorded in the 1990s [42]. Serological investigation of malaria antibodies in these provinces in 2006 and 2007 showed that about $87.83 \%$ of the positive samples were asymptomatic cases [43], indicating that existing control measures were failing to protect these populations, since then the infection source of vivax malaria has been accumulating and increasing significantly.

For the vector capacity, human blood index (HBI) is an important and quantitative indicator, which showed the contact relationship between mosquitoes and humans [17]. HBI of the areas where An. sinensis was present was about $5 \%$ in the 1990 s, which was attributed to the reduction in the incidence of vivax malaria in the region [44]. Reviewing the data during the 1990s, the number of pigs and farm cattle reared by each family increased, which caused the mosquitoes to feed more on blood from livestock instead of human blood. But since the development of the economy and popularity of agricultural mechanization, the farmers no longer keep pigs in their houses, and the number of farm cattle has also been greatly reduced. The increased probability of An. sinensis feeding on human blood indicates that the mosquitoes change their behavior caused by changes in number of livestock [45,46]. In addition, our study also revealed that the HBIs in Huaiyuan and Yongcheng were 0.6667 and 0.6429 , and the HBI values were more than 12 times higher than that in the historical records. Results in the study also indicated that the increasing HBI value was due to the changes of human activities or living customs [25,47].

It has been observed that the local villagers generally used mosquito-nets and mosquito-repellent incense when asleep, and most families have screened-doors, and/ or screened-windows. Only 7 out of 81 mosquito nets had Anopheles spp. mosquitoes inside nets in the morning of the investigation, which indicated that the chance of contact between mosquitoes and humans might occur. However, the mosquito-biting was inevitable because the villagers had a habit of enjoying cool outdoor fresh air before midnight in the Summer time [2].

The average man-biting rate observed in the 1990s in the areas where $A n$. sinensis was the only vector was 2.36 [48], however, the rates in the study sites were 6.0957 and 5.8621 according to this investigation, which is more than 2.5 times higher than before. From the history data, the average vector capacity was 0.331 in the 1990s in Huanghuai valley where An. sinensis was mostly predominately distributed [48], and was 0.1686 during 1996-1998 in Henan province [2,38]. However, our findings showed that the vector capacity was 0.7740 and 0.5502 in Anhui and Henan province, respectively, about 2.3 and 1.7 times higher than that in the 1990s, and were 4.6 and 3.3 times higher than that in Henan during 1996-1998, respectively. All these results demonstrated that the ability of An. sinensis to transmit $P$. vivax had been obviously enhanced; therefore, more effective and practical control measures need to be implemented in Huanghuai valley, particularly in the mosquito control and reduction of the residual carriers with $P$. vivax. Our findings illustrated that the vector capacity is a good indicator to assess the malaria transmission capability, which contributes to changes in transmission patterns of vivax malaria.

\section{Conclusions}

This study suggests that vivax malaria outbreaks in Huanghuai valley is highly related to the enhancement in transmission ability for P. vivax through An. sinensis, which is attributed to the habits of local residents and the remarkable drop in the number of large livestock leading to disappearance of traditional biological barriers.

\section{Competing interests}

The authors declare that they have no competing interests.

\section{Authors' contributions}

Conceived and designed the experiments: JYP, SSZ, XZ, FH. Performed the experiments: XZ, FH, DQW, MZS, YPS, GCZ, JJJ. Analyzed the data: JYP, SSZ, $X Z$. Contributed reagents/materials/analysis tools: JYP, SSZ, XZ, FH. Wrote the paper: JYP, SSZ, XZ. All authors read and approved the final version of the manuscript. 


\section{Financial support}

The study was funded through the National S \& T Mayor Project (Grant no.2008ZX10004-011, 2012ZX10004-22).

\section{Acknowledgements}

The study participants are gratefully acknowledged for their collaboration and the staff at Anhui Center for Disease Control and Prevention, and Henan Center for Disease Control and Prevention. Thanks to Professor Xiao-Nong Zhou and Dr. Ernest Tambo from the National Institute of Parasitic Diseases, China CDC for improving the manuscript and Dr. Kun Yang for drawing the location map.

\section{Author details}

${ }^{1}$ National Institute of Parasitic Diseases, Chinese Center for Disease Control and Prevention, Shanghai 200025 People's Republic of China. ${ }^{2} \mathrm{WHO}$ Collaborating Centre for Malaria, Schistosomiasis and Filariasis, Key Laboratory of Parasite \& Vector Biology, Ministry of Health, Shanghai 200025 People's Republic of China. ${ }^{3}$ Anhui Center for Disease Control and Prevention, Wuhu 241000 People's Republic of China. ${ }^{4}$ Henan Center for Disease Control and Prevention, Zhengzhou 450016 People's Republic of China. ${ }^{5}$ Yuangcheng Center for Disease Control and Prevention, Yuangchen, Henan province 450000 People's Republic of China. ${ }^{6}$ Yongqiao District Center for Disease Control and Prevention, Shuzhou, Anhui province 241000 People's Republic of China.

Received: 20 June 2012 Accepted: 29 June 2012

Published: 9 July 2012

\section{References}

1. Ning $X$, Qin $L$, Jinchuan $Y$, Jiuping $Y$, Xintian $L$ : Surveillance of risk factors from imported cases of falciparum malaria in Sichuan, China. Southeast Asian J Trop Med Public Health 1999, 30:235-239.

2. Zhou SS, Huang F, Wang JJ, Zhang SS, Su YP, Tang LH: Geographical, meteorological and vectorial factors related to malaria re-emergence in Huang-Huai River of central China. Malaria J 2010, 9:337.

3. Zhou SS, Zhang SS, Wang JJ, Zheng X, Huang F, Li WD, Xu X, Zhang HW: Spatial correlation between malaria cases and water-bodies in Anopheles sinensis dominated areas of Huang-Huai plain China. Parasit Vectors 2012, 5:106.

4. Sleigh $A C$, Liu XL, Jackson S, Li P, Shang LY: Resurgence of vivax malaria in Henan Province, China. Bull World Health Organ 1998, 76:265-270.

5. Qian HL, Tang LH: The achievement and the outlook of 50 years malaria prevention and cure work of China. Chin J Epi 2000, 21:225-227. in Chinese.

6. Tang L: Progress in malaria control in China. Chin Med J (Engl) 2000, 113:89-92.

7. Hui FM, Xu B, Chen ZW, Cheng X, Liang L, Huang HB, Fang LQ, Yang H, Zhou HN, Yang HL, Zhou XN, Cao WC, Gong P: Spatio-temporal distribution of malaria in Yunnan Province, China. AmJTrop Med Hyg 2009, 81:503-509.

8. Yang GJ, Gao Q, Zhou SS, Malone JB, McCarroll JC, Tanner M, Vounatsou P, Bergquist $\mathrm{R}$, Utzinger J, Zhou XN: Mapping and predicting malaria transmission in the People's Republic of China, using integrated biologydriven and statistical models. Geospat Health 2010, 5:11-22.

9. Huang F, Zhou S, Zhang S, Zhang H, Li W: Meteorological factors-based spatio-temporal mapping and predicting malaria in central China. AmJTrop Med Hyg 2011, 85:560-567.

10. Wen L, Li C, Lin M, Yuan Z, Huo D, Li S, Wang Y, Chu C, Jia R, Song H: Spatio-temporal analysis of malaria incidence at the village level in a malaria-endemic area in Hainan China. Malaria J 2011, 10:88.

11. Zhou SS, Tang LH, Sheng HF, Wang Y: [Malaria situation in the People's Republic of China in 2004]. Zhongguo Ji Sheng Chong Xue Yu Ji Sheng Chong Bing Za Zhi 2006, 24:1-3. in Chinese.

12. Liu XB, Liu QY, Guo YH, Jiang JY, Ren DS, Zhou GC, Zheng CJ, Zhang Y, Liu JL, Li ZF, Chen Y, Li HS, Morton LC, Li HZ, Li Q, Gu WD: The abundance and host-seeking behavior of culicine species (Diptera: Culicidae) and Anopheles sinensis in Yongcheng city People's Republic of China. Parasit Vectors 2011, 4:221.
13. Zhou SS, Wang Y, Fang W, Tang LH: [Malaria situation in the People's Republic Of China in 2007]. Zhongguo Ji Sheng Chong Xue Yu Ji Sheng Chong Bing Za Zhi 2008, 26:401-403. in Chinese.

14. Zhou SS, Tang LH, Sheng HF: [Malaria situation in the People's Republic of China in 2003]. Zhongguo Ji Sheng Chong Xue Yu Ji Sheng Chong Bing Za Zhi 2005, 23:385-387. in Chinese.

15. Gu ZC, Shang LY, Chen JS, Zheng X, Su YJ, Li AM, Liu H, Luo MZ, Qian HL, Tang LH: [The role of Anopheles anthropophagus in malaria transmission in in Xinyang City of Henan Province]. Zhongguo Ji Sheng Chong Xue Yu Ji Sheng Chong Bing Za Zhi 2001, 19:221-224. in Chinese.

16. Qian $H$, Tang $L$, Cheng $Y$, Yang B: [Preliminary estimation of malaria transmission potential in areas where Anopheles sinensis is the only vector]. Zhongguo Ji Sheng Chong Xue Yu Ji Sheng Chong Bing Za Zhi 1994, 12:265-267. in Chinese.

17. Liu C: [Comparative studies on the role of Anopheles anthropophagus and Anopheles sinensis in malaria transmission in China]. Zhonghua Liu Xing Bing Xue Za Zhi 1990, 11:360-363. in Chinese.

18. Liu XB, Liu QY, Guo YH, Jiang JY, Ren DS, Zhou GC, Zheng CJ, Liu JL, Chen Y, Li HS, Li HZ, Li Q: Random repeated cross sectional study on breeding site characterization of Anopheles sinensis larvae in distinct villages of Yongcheng City People's Republic of China. Parasit Vectors 2012, 5:58.

19. Paaijmans KP, Blanford S, Chan BH, Thomas MB: Warmer temperatures reduce the vectorial capacity of malar Discussionia mosquitoes. Biol Lett 2012, 8:465-468.

20. Sinka ME, Bangs MJ, Manguin S, Chareonviriyaphap T, Patil AP, Temperley WH, Gething PW, Elyazar IR, Kabaria CW, Harbach RE, Hay SI: The dominant Anopheles vectors of human malaria in the Asia-Pacific region: occurrence data, distribution maps and bionomic precis. Parasit Vectors 2011, 4:89.

21. Zhou SS, Wang Y, Xia ZG: [Malaria situation in the People's Republic Of China in 2009]. Zhongguo Ji Sheng Chong Xue Yu Ji Sheng Chong Bing Za Zhi 2011, 29:1-3. in Chinese.

22. Qi G: [Opportunities and challenges of malaria elimination in China]. Zhongguo Xue Xi Chong Bing Fang Zhi Za Zhi 2011, 23:347-349. in Chinese.

23. WHO: Malaria vector control and personal protection: report of a WHO study group, Series. Geneva: World Health Organization; 2006

24. RBM: Key facts, figures and strategies: the Global Malaria Action Plan.: Roll Back Malaria Partnership; 2008. http://www.rbm.who.int/toolbox/tool_GMAP. html.

25. Cohuet A, Harris C, Robert V, Fontenille D: Evolutionary forces on Anopheles: what makes a malaria vector? Trends Parasitol 2010, 26:130-136.

26. Garrett-Jones C: Prognosis for Interruption of Malaria Transmission through Assessment of the Mosquito's Vectorial Capacity. Nature 1964, 204:1173-1175.

27. Paaijmans KP, Blanford S: Chan BH. Thomas MB: Warmer temperatures reduce the vectorial capacity of malaria mosquitoes. Biol Lett; 2011.

28. Cui L, Yan G, Sattabongkot J, Chen B, Cao Y, Fan Q, Parker D, Sirichaisinthop J, Su XZ, Yang H, Yang Z, Wang B, Zhou G: Challenges and prospects for malaria elimination in the Greater Mekong Subregion. Acta Trop 2011, 121:240-245.

29. Garrett-Jones C, Shidrawi GR: Malaria vectorial capacity of a population of Anopheles gambiae: an exercise in epidemiological entomology. Bull World Health Organ 1969, 40:531-545.

30. Vythilingam I, Phetsouvanh R, Keokenchanh K, Yengmala V, Vanisaveth V, Phompida S, Hakim SL: The prevalence of Anopheles (Diptera: Culicidae) mosquitoes in Sekong Province, Lao PDR in relation to malaria transmission. Trop Med Int Health 2003, 8:525-535.

31. Jjumba JN, Mosha FW, Lindsay SW: Malaria transmission risk variations derived from different agricultural practices in an irrigated area of northern Tanzania. Med Vet Entomol 2002, 16:28-38.

32. Ree HI, Hwang UW, Lee IY, Kim TE: Daily survival and human blood index of Anopheles sinensis, the vector species of malaria in Korea. J Am Mosq Control Assoc 2001, 17:67-72

33. Luo MZ, Zheng X, Chen CY, et al: Comparison of three survey methods for testing blood preference of Anopheles sinensis in Wuxue City. Chin J Parasit Dis Contrl 1994, 7:219-221. in Chinese.

34. Kere NK, Arabola A, Bakote'e B, Qalo O, Burkot TR, Webber RH, Southgate BA: Permethrin-impregnated bednets are more effective than DDT house-spraying to control malaria in Solomon Islands. Med Vet Entomol 1996, 10:145-148. 
35. Molineaux L, Dietz K, Thomas A: Further epidemiological evaluation of a malaria model. Bull World Health Organ 1978, 56:565-571.

36. Dietz K, Molineaux L, Thomas A: A malaria model tested in the African savannah. Bull World Health Organ 1974, 50:347-357.

37. Khan $A Q$, Talibi SA: Epidemiological assessment of malaria transmission in an endemic area of East Pakistan and the significance of congenital immunity. Bull World Health Organ 1972, 46:783-792.

38. Qu CZ, Su TZ, Wang MY, et al: Vectorial capacity of malaria transmission of Anopheles sinensis in Zhengzhou in nature. J Henan Med Uni 2000, 35:394-396. in Chinese.

39. Control TmCGoV: A research agenda for malaria eradication: vector control. PLoS Med 2011, 8:e1000401.

40. Tyagi BK: A review of the emergence of Plasmodium falciparumdominated malaria in irrigated areas of the Thar Desert, India. Acta Trop 2004, 89:227-239.

41. Liu XZ, Xu BL: [Malaria situation and evaluation on the control effect in Henan Province during 1990-2005]. Zhongguo Ji Sheng Chong Xue Yu Ji Sheng Chong Bing Za Zhi 2006, 24:226-229. in Chinese.

42. Qian HL: Malaria Situation in the People's Republic of China in 1993. Chin J Parasitol Parasitic Dis 1994, 12:161-164. in Chinese.

43. Zheng $X$, Tang $L H$, Gu ZC, Zhu TH, Shi WQ, Jiang WK, Zhou SS, Pan B, Lin RX: [Morphology and habits of An. anthropophagus and its role in malaria transmission in Hengqin Island of Zhuhai City]. Zhongguo $\mathrm{Ji}$ Sheng Chong Xue Yu Ji Sheng Chong Bing Za Zhi 2007, 25:488-491. in Chinese.

44. Qian HL, Deng D, Guan DH, et al: Investigation and quantitative analysis of the components of vectorial capacity of Anopheles sinensis. Chin J Parasltol Parasit Dis 1984, 2:3. in Chinese.

45. Habtewold T, Prior A, Torr SJ, Gibson G: Could insecticide-treated cattle reduce Afrotropical malaria transmission? Effects of deltamethrin-treated Zebu on Anopheles arabiensis behaviour and survival in Ethiopia. Med Vet Entomol 2004, 18:408-417.

46. Wu KC, Chen WJ, Wang ZG, Cai XZ, Deng D, Hu LK, Liu ZY, Zhu WG, Guan DH, Jiang WK, et al: [Studies on distribution and behavior of Anopheles minimus and its role of malaria transmission in Hainan Province at present]. Zhongguo Ji Sheng Chong Xue Yu Ji Sheng Chong Bing Za Zhi 1993, 11:120-123. in Chinese.

47. Hancock PA, Thomas MB, Godfray HC: An age-structured model to evaluate the potential of novel malaria-control interventions: a case study of fungal biopesticide sprays. Proc Biol Sci 2009, 276:71-80.

48. Qian $H L$, Tang $L H$, Tang $L Y$, et al: Preliminary estimation on the critical value of man biting rate and vectorial capacity of Anopheles sinensis. Practical Preventire Med 1996, 3:1-2. in Chinese.

\section{Submit your next manuscript to BioMed Central and take full advantage of:}

- Convenient online submission

- Thorough peer review

- No space constraints or color figure charges

- Immediate publication on acceptance

- Inclusion in PubMed, CAS, Scopus and Google Scholar

- Research which is freely available for redistribution 